\title{
Theoretical Chemistry Symposium 2006
}

\section{Foreword}

This special issue of the Journal of Chemical Sciences contains 29 original papers presented at the Theoretical Chemistry Symposium (TCS 2006) held at Bharathidasan University during December 11-13, 2006. Earlier meetings on this theme were organized in various parts of the country viz. Chandigarh (1986), IIT Madras (1990), IIT Khargapur (1993), University of Pune \& NCL 1998, M.G. University, Kottayam (1996), University of Hyderabad (1998), IIT, Kanpur (2000), IACS, Kolkata (2003) and BARC, Mumbai (2004). The Tiruchirappalli meeting formed part of the Silver Jubilee celebrations of the Bharathidasan University.

Theoretical Chemistry is experiencing rapid growth in recent years and has become multifaceted. Development of newer methodologies and novel applications in materials, life sciences and other areas have contributed to this enormous growth. The papers presented in this issue cover several important areas such as Theoretical \& Computational Methodologies, Density Functional \& Wave function based Quantum Chemistry, Statistical Mechanics \& its Applications, Classical \& Quantum Simulation of Complex Systems, Electronic Structure \& Spectroscopy of Molecules \& Clusters, Chemical Dynamics, Reactivity \& Catalysis, Chemistry at Surfaces and Interfaces, Nonlinear Phenomena \& Dynamics, Molecular Materials \& Nano-Systems, Soft Condensed Matter, Biology-inspired Theoretical Chemistry, Equilibrium \& Non-equilibrium Processes in Condensed Phase, Chemo \& Bioinformatics and Modelling.

We thank all the authors for their valuable contributions. We would like to express our sincere thanks to all the reviewers for their cooperation and timely help and $\mathrm{Mr}$ Senthil and Jaccob for their administrative help. We also thank the Editor Journal of Chemical Sciences and the Editorial Board of the Indian Academy of Sciences for agreeing to bring out this Special issue. Our sincere thanks are due to the Editorial Staff for their tireless efforts.

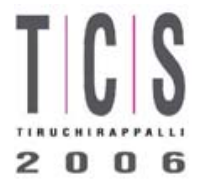

SOURAV PAL

$P$ VENUVANALINGAM

(Guest Editors) 